\title{
Redefining Readmission Risk Factors for General Medicine Patients
}

Nazima Allaudeen, $\mathrm{MD}^{1,2}$
Arpana Vidyarthi, $\mathrm{MD}^{2}$
Judith Maselli, $\mathrm{MSP}^{2}$
Andrew Auerbach, $\mathrm{MD}, \mathrm{MPH}^{2}$

\author{
${ }^{1}$ Department of Medicine, Veterans Affairs (VA) Healthcare System, Palo Alto, California. \\ ${ }^{2}$ Department of Medicine, Division of Hospital Medicine, University of California San Francisco, \\ San Francisco, California.
}

Disclosure: Nothing to report.

BACKGROUND: Readmissions are costly both financially for our healthcare system and emotionally for our patients. Identifying factors that increase risk for readmissions may be helpful to focus resources to optimize the discharge process and reduce avoidable readmissions.

OBJECTIVE: To identify factors associated with readmission within 30 days for general medicine patients.

METHODS: We performed a retrospective observational study of an administrative database at an urban 550-bed tertiary care academic medical center. Cohort patients were discharged from the general medicine service over a 2-year period from June 1, 2006, to May 31, 2008. Clinical, operational, and sociodemographic factors were evaluated for association with readmission. RESULTS: Our cohort included 10,359 consecutive admissions (6805 patients) discharged from the general medicine service. The 30 -day readmission rate was $17.0 \%$. In multivariate analysis, factors associated with readmission included black race (odds ratio [OR], 1.43; 95\% confidence interval [CI], 1.24-1.65), inpatient use of narcotics (1.33; 1.16-1.53) and corticosteroids (1.24; 1.09-1.42), and the disease states of cancer (with metastasis 1.61 ; 1.33-1.95; without metastasis 1.95; 1.54-2.47), renal failure (1.19; 1.05-1.36), congestive heart failure (1.30; 1.09-1.56), and weight loss (1.26; 1.09-1.47). Medicaid payer status (1.15; 0.97-1.36) had a trend toward readmission.

CONCLUSION: Readmission of general medicine patients within 30 days is common and associated with several easily identifiable clinical and nonclinical factors. Identification of these risk factors can allow providers to target interventions to reduce potentially avoidable readmissions. Journal of Hospital Medicine 2011;6:54-60. ㅇ 2011 Society of Hospital Medicine

\section{KEYWORDS: general medicine, readmission, risk factors, transitions in care.}

Within Medicare recipients, an astounding one in five medical patients $(19.6 \%)$ is readmitted within 30 days, accounting for $\$ 15$ billion in spending. ${ }^{1,2}$ Amidst the current healthcare system crisis, reducing these hospital readmissions has risen to the highest priority. Reducing readmissions is the newest addition to multiple quality dashboards, both institutional and national, as a measure of the care delivered during hospitalization. ${ }^{3}$ One of the most notable of these reporting entities, Hospital Compare, now publicly reports Medicare readmission rates for a few common diagnoses. ${ }^{4}$ While Medicare already withholds payment to hospitals for readmissions within 24 hours for the same diagnosis, Medicare may soon reduce payment to hospitals with the highest rates of readmission within 30 days, a powerful incentive for hospitals to intervene. Readmissions have also reached the radar of additional stakeholders, even making its way onto Obama's budget considerations, given the potential cost savings to the system overall. ${ }^{5}$

To develop systems which reduce readmissions, one must first gain understanding of the characteristics of readmissions. A few clinical risk factors (such as age, number of prior admissions, and comorbidities) have been well defined in subgroups of general medicine inpatients. ${ }^{6-12}$ Likewise, interventions aiming to reduce readmissions have also focused on subgroups, excluding a large portion of hospitalized patients (for example, non-English speakers and younger patients). ${ }^{13-20}$ Other data have been derived in veterans or within non-US populations that have inherently different payer, race, ethnicity, and primary language composition, and may not be applicable outside those settings. ${ }^{7,8,10,11,21}$ Lastly, little is known regarding risk that may be associated with operational factors, such as weekend discharge or admission source. As a result, there are few data describing the clinical, operational, and demographic factors associated with readmission in a heterogeneous population of hospitalized general medicine patients-the patient population of most generalists in the United States.

To understand the impact of a variety of risk factors in a diverse general medicine population, we evaluated the characteristics of readmitted patients in a large urban university medical center over a 2-year period. We hypothesized that a number of clinical, operational, and sociodemographic factors would be associated with readmission.

\section{Methods}

\section{Sites and Subjects}

Our data were collected on general medicine patients during hospitalization between June 1, 2006 and May 31, 2008, at the University of California San Francisco. The University of California, San Francisco (UCSF) Medical Center is composed of Moffitt-Long Hospital (a 400-bed center) and 
UCSF-Mount Zion Hospital (a 200-bed facility) located in San Francisco, CA.

Medical patients at Moffitt-Long Hospital are admitted to 1 of 8 medical teams composed of a resident, 1 to 2 interns, and 0 to 3 medical students, supervised by an attending physician who is most often a hospitalist. At Moffitt-Long Hospital, housestaff write all orders and provide 24-hour coverage to inpatients. Mount Zion medical patients are cared for by 1 of 2 teams and staffed by a hospitalist on each team who is responsible for all elements of care. Both services care for common inpatient diagnoses, as well as specialty-associated diagnoses such as cancer, pneumonia, and chronic obstructive pulmonary disease (COPD). Of note, at Moffitt-Long Hospital, those patients with primary cardiac diagnoses are cared for by a separate team composed of housestaff and students supervised by a cardiologist.

The discharge process at both sites utilizes a multidisciplinary team-including physicians, case managers, nurses, pharmacists, and discharge coordinators-working in concert. Key components include arranging follow-up care, faxing the discharge summary to the primary care provider, and educating the patient and caregivers, especially regarding medications. While these goals are clearly delineated, significant variability exists in how these tasks are actually accomplished. The multidisciplinary approach, components of the discharge process, and lack of a systematic approach are representative of the discharge process around the country. ${ }^{22}$

\section{Data}

Data regarding patient demographics, age, comorbidities, and insurance status were collected from administrative data systems at UCSF, reflecting the patient's status at the time of index admission. These same systems were used to collect a date-stamped log of all medications (eg, anticoagulants) for which the patient was billed during the last 48 hours of hospitalization. Specifically, data were obtained for medications previously shown to cause adverse drug events following hospital discharge. ${ }^{23,24}$ These medication groups include corticosteroids, anticoagulants, antibiotics, narcotics, nonsteroidal anti-inflammatory drugs (NSAIDs), cardiovascular medications, antiepileptics, anticholinergics, antidepressants, and antidiabetics. Operational factors that we hypothesized would affect readmission risk included admission source, discharge disposition, and weekday vs. weekend discharge. Case management, social work, and pharmacy services operate with limited staffing on weekends. Likewise, resident and intern physicians are more likely to be off on a weekend day than a weekday; covering attending physicians care for about half of patients during the weekend. Data were obtained from Transition Systems International (TSI, Boston, MA) administrative databases, a cost-accounting system that collects data abstracted from patient charts upon discharge from UCSF.

\section{Definition of Readmission Measure}

Using TSI, we detected readmission to UCSF by screening for any inpatient encounters on any service (not just medicine) within the 30 days following discharge from the general medicine service at the 2 UCSF campuses. We excluded elective readmissions, such as for scheduled chemotherapy. Patients who died at the index admission were excluded from the cohort.

\section{Adjustment Variables}

Age, gender, payer status and APR risk of mortality (3M Health Information Systems, St. Paul, MN) were collected from administrative data. The All Patient Refined (APR) risk of mortality is the "all patients risk" of mortality score developed by $3 \mathrm{M}$ which divides patients into 4 subclasses of risk based on clinical problems and comorbidities. ${ }^{25}$ We used secondary diagnosis codes in administrative data to classify comorbidities using the method of Elixhauser. ${ }^{26}$

Using the log of medication charges, we grouped highrisk medications according to the classification scheme of Forster et al. ${ }^{23}$ and Hanlon et al. ${ }^{24}$ We then created a count representing the total number of medications administered to patients within the final 48 hours of stay.

\section{Analysis}

We first described study patients and hospitals using univariable methods. Multivariable generalized estimating equations (SAS PROC GENMOD) were used to account for clustering of patients within physicians and calculate adjusted odds ratios (ORs). As there were 2 sites within UCSF Medical Center (Moffitt-Long and Mount Zion hospitals), we included site as a fixed effect in our model. Models were constructed using manual variable selection methods with final selection being made based on whether the covariate was associated with readmission at $P<0.05$. All analyses were carried out using SAS version 9.2 (SAS Institute, Inc. Cary, NC).

\section{Results}

\section{Baseline Characteristics}

During the 2-year accrual period, 295 attending physicians admitted 6805 unique patients for a total of 10,359 admissions. Seventeen percent of these 10,359 admissions were readmitted within 30 days. The cohort of all patients had a mean age of 59.6 years \pm 19.5 standard deviation (SD), with $52.8 \%$ women. The mean length of stay was 5.6 days \pm 10.4 SD. Medicare was the payer source for approximately half of the admissions. The majority of admissions $(90.4 \%)$ were billed for at least 1 high risk medication, with narcotics, cardiac medications, and antibiotics being the most common. Regarding disposition, $79.5 \%$ of admissions were discharged to home; $9.1 \%$ were discharged to a skilled nursing facility (SNF).

Baseline sociodemographic, operational, and clinical characteristics for patients readmitted and not readmitted 
are shown in Table 1. Demographic characteristics with significant differences $(P<0.05)$ between readmitted and nonreadmitted groups included mean age, race, payer status, and primary language other than English. Regarding operational characteristics, readmitted patients had a higher median length of stay and were more likely to be admitted through the emergency room during their index admission. Discharge to an SNF was higher in the readmitted group versus the nonreadmitted group (9.7\% vs. 9.0\%). Several clinical factors were more prevalent in the readmitted group: high-risk medications, specifically steroids, narcotics, and cardiovascular medications; high-risk medication count of 3 or greater; and comorbidities including congestive heart failure, renal disease, cancer, anemia, and depression.

\section{Frequency of Readmission}

The 30-day readmission rate was $17.0 \%$ (1762 patients), with $49.7 \%$ (875 patients) of the readmissions occurring within 10 days of discharge. Of patients readmitted, the general medicine service was the readmitting team in $78.2 \%$. A quarter of readmissions $(26.2 \%)$ had the same primary diagnosis on initial and repeat admission.

\section{Factors Associated With Readmission}

Factors associated with readmission were categorized as sociodemographic, operational, and clinical. Factors associated with readmission with $P<0.05$ and present in at least $5 \%$ of admissions are presented in Table 2. Of the sociodemographic factors, black race was significantly associated with readmission. Within the Medicare cohort, risk for readmission was similar for white vs. nonwhite race, with relative risk of 1.0 (95\% confidence interval [CI], 0.86-1.18). Medicaid as payer status was significantly associated in the unadjusted model, and in the adjusted model showed a trend toward readmission. Mean age was significantly different in the readmitted and nonreadmitted groups, but the difference was small (1.0 year). Moreover, when we evaluated age in 5-year categories (ex. 65-70, 71-75, etc.), age was not associated with readmission. In the adjusted model, none of the operational factors were significantly associated with readmission, including discharge to SNF, weekend discharge, or admit source.

Of the clinical factors, high-risk medications and 6 comorbidities were associated with readmission. High-risk medication categories associated with readmission were steroids and narcotics; anticholinergics medications were protective. The 6 comorbidities associated with readmission were congestive heart failure, renal disease, cancer (with and without metastasis), weight loss, and iron deficiency anemia. While APR risk of mortality was associated with readmission at $P<0.05$, including APR in our final model did not alter which other factors were significantly associated with readmission. When site (Moffitt-Long vs. Mount Zion Hospitals) was added to the model, the ORs for factors associated with readmission did not change appreciably $( \pm 0.01)$.

\section{Discussion}

In this retrospective observational study of hospitalized patients, we found that readmission was common and associated with a number of risk factors that could be easily identified early in hospitalization. Nonclinical factors associated with readmission were black race and Medicaid payer status (in the unadjusted model). Clinical factors were high risk medications including steroids and narcotics; and comorbidities including congestive heart failure, renal disease, cancer, anemia, and weight loss. In contrast, other potential risks-such as discharge on a weekend and discharge to an SNF-were not independently associated with readmission. This cohort-with a mix of clinical scenarios, payers, age, etc.-represents the inherently heterogeneous population of inpatient general medicine across the country and abroad. Hospitalists provided care for over $65 \%$ of the general medicine service, again representative of the trend in US inpatient medicine. ${ }^{27,28}$ Lastly, our cohort did not have the benefit of a systematic and consistent discharge process with interventions focused on reducing readmissions. This gap, which is common across hospitals, highlights the utility of this data in targeting quality improvement efforts.

Reducing risks for readmission requires identification of patient populations at highest risk; in those patients, one can further identify factors which are potentially modifiable via education or patient-engagement interventions. While in the hospital, more intensive predischarge counseling and efforts to increase mobility may be most useful if targeted early and often on those at highest risk. ${ }^{15,16,29,30}$ Finally, broader-based support in the form of better home services, more access to longitudinal care, or targeted postdischarge efforts may be required. ${ }^{14,31}$

Though current strategies focus largely on clinical risk factors, this study shows that nonclinical factors play an equally important but underappreciated role in contributing to readmission. While prior studies have shown variable results on association of black race with readmission, ${ }^{2,9,11}$ none have evaluated or linked Medicaid to readmission, which just missed statistical significance in this study (OR, 1.15; 95\% CI, 0.97-1.36). Both black race and Medicaid as payer are proxies for the underlying root cause aspects leading to readmission, such as access to longitudinal care. Following this trail to the "root cause" will require in-depth qualitative evaluation that includes the patient perspective as a source of data. ${ }^{32}$ For example, risk for readmission may not stem solely from being on warfarin, but in combination with not having transportation to get an international normalized ratio (INR) checked, a suboptimal understanding of how to take the medication, or not recognizing potential side effects until too late to avoid inpatient admission. 


\begin{tabular}{|c|c|c|c|}
\hline Characteristic & $\begin{array}{l}\text { Patients Readmitted } \\
(\mathrm{n}=176217.0 \%), \mathrm{n}(\%)\end{array}$ & $\begin{array}{l}\text { Patients Not Readmitted } \\
(\mathrm{n}=859783.0 \%), \mathrm{n}(\%)\end{array}$ & $P$ Value \\
\hline Mean age (years) (SD) & $58.8(19.3)$ & $59.8(19.6)$ & 0.0491 \\
\hline Female & $930(52.8)$ & $4548(52.9)$ & 0.9260 \\
\hline \multicolumn{4}{|l|}{ Race* $^{*}$} \\
\hline White & $785(44.6)$ & 4166 (48.8) & \multirow[t]{4}{*}{$<0.0001$} \\
\hline Black & $442(25.1)$ & $1401(16.4)$ & \\
\hline Asian & $323(18.4)$ & $1726(20.2)$ & \\
\hline Other & $209(11.9)$ & $1240(14.5)$ & \\
\hline Hispanic ethnicity ${ }^{\dagger}$ & $140(8.1)$ & $734(8.9)$ & 0.2737 \\
\hline \multicolumn{4}{|l|}{ Payer status } \\
\hline Medicare & $905(51.4)$ & $4266(49.6)$ & \multirow[t]{4}{*}{$<0.0001$} \\
\hline Medicaid/Medi-cal & $458(26.0)$ & $1578(18.4)$ & \\
\hline Private & $370(21.0)$ & $2092(24.3)$ & \\
\hline Other & $29(1.7)$ & $661(7.7)$ & \\
\hline Primary language other than English ${ }^{\ddagger}$ & $242(17.1)$ & 1394 (19.5) & 0.0359 \\
\hline Median length of stay (days) (interquartile range) & $4(2,7)$ & $3(2,6)$ & $<0.0001$ \\
\hline \multicolumn{4}{|l|}{ Admit source } \\
\hline Emergency room & $1506(85.5)$ & $6898(80.2)$ & \multirow[t]{3}{*}{$<0.0001$} \\
\hline Outside hospital & $38(2.2)$ & $271(3.2)$ & \\
\hline Direct admission/other (jail) & $218(12.4)$ & $1428(16.6)$ & \\
\hline \multicolumn{4}{|l|}{ Discharge to } \\
\hline Home & $1461(82.9)$ & $6773(78.8)$ & \multirow[t]{3}{*}{$<0.0001$} \\
\hline SNF & $170(9.7)$ & $774(9.0)$ & \\
\hline Other & $131(7.4)$ & $1050(12.2)$ & \\
\hline Discharged on weekend & $381(21.6)$ & $1904(22.1)$ & 0.6288 \\
\hline \multicolumn{4}{|l|}{ Patient medications } \\
\hline Any high-risk medication ${ }^{\S}$ & 1679 (95.3) & $7684(89.4)$ & $<0.0001$ \\
\hline \multicolumn{4}{|l|}{ High-risk medication count } \\
\hline $0-2$ & $577(32.8)$ & $3666(42.6)$ & \multirow[t]{3}{*}{$<0.0001$} \\
\hline $3-4$ & $692(39.3)$ & $2968(34.5)$ & \\
\hline$\geq 5$ & $493(28)$ & $1963(22.8)$ & \\
\hline Any corticosteroids & $399(22.6)$ & $1571(18.3)$ & $<0.0001$ \\
\hline Anticoagulant & $120(6.8)$ & $559(6.5)$ & 0.6340 \\
\hline Any antibiotic & $904(51.3)$ & $4203(48.9)$ & 0.0646 \\
\hline Any narcotic & $1036(58.8)$ & $4206(48.9)$ & $<0.0001$ \\
\hline Any NSAID & $68(3.9)$ & $320(3.7)$ & 0.7826 \\
\hline Any cardiovascular med & $887(50.3)$ & 3806 (44.3) & $<0.0001$ \\
\hline Any antiepileptic & $93(5.3)$ & $470(5.5)$ & 0.7500 \\
\hline Any anticholinergic & $47(2.7)$ & $354(4.1)$ & 0.0040 \\
\hline Any antidepressant & $455(25.8)$ & $1863(25.8)$ & 0.0001 \\
\hline Any antidiabetic & $198(11.2)$ & 994 (11.6) & 0.6970 \\
\hline \multicolumn{4}{|l|}{ Elixhauser comorbidities } \\
\hline Congestive heart failure & $219(12.4)$ & $795(9.3)$ & $<0.0001$ \\
\hline Pulmonary circulation disease & $72(4.1)$ & $178(2.1)$ & $<0.0001$ \\
\hline Peripheral vascular disease & $84(4.8)$ & $331(3.9)$ & 0.0737 \\
\hline Hypertension & $745(42.3)$ & $3741(43.5)$ & 0.3411 \\
\hline Other neurological disease & $101(5.7)$ & $696(8.1)$ & 0.0007 \\
\hline Chronic pulmonary disease & $317(18.0)$ & $1442(16.8)$ & 0.2149 \\
\hline Diabetes & $303(17.2)$ & $1333(15.5)$ & 0.0762 \\
\hline Renal failure & $339(19.2)$ & $1286(15.0)$ & $<0.0001$ \\
\hline Liver disease & $188(10.7)$ & $774(9.0)$ & 0.0281 \\
\hline Metastatic cancer & $160(9.1)$ & $530(6.2)$ & $<0.0001$ \\
\hline Solid tumor w/o metastases & $100(5.7)$ & $277(3.2)$ & $<0.0001$ \\
\hline Rheumatoid arthritis/collagen vas & $81(4.6)$ & $303(3.5)$ & 0.0299 \\
\hline Weight loss & $153(8.7)$ & $584(6.8)$ & 0.0449 \\
\hline Deficiency anemia & $522(29.6)$ & $1979(23.0)$ & $<0.0001$ \\
\hline Alcohol abuse & $101(5.7)$ & $428(5.0)$ & 0.1905 \\
\hline Drug abuse & $148(8.4)$ & $619(7.2)$ & 0.0798 \\
\hline Depression & $244(13.9)$ & $963(11.2)$ & 0.0016 \\
\hline
\end{tabular}


TABLE 1. (Continued)

\begin{tabular}{|c|c|c|c|}
\hline Characteristic & $\begin{array}{l}\text { Patients Readmitted } \\
(\mathrm{n}=176217.0 \%), \mathrm{n}(\%)\end{array}$ & $\begin{array}{l}\text { Patients Not Readmitted } \\
(\mathrm{n}=859783.0 \%), \mathrm{n}(\%)\end{array}$ & $P$ Value \\
\hline \multicolumn{4}{|c|}{ APR risk of mortality } \\
\hline 1 & $451(25.6)$ & 3101 (36.1) & $<0.0001$ \\
\hline 2 & 619 (35.1) & 2797 (32.5) & \\
\hline 3 & 536 (30.4) & $1907(22.2)$ & \\
\hline 4 & $156(8.9)$ & $792(9.2)$ & \\
\hline
\end{tabular}

Abbreviations: CHF, congestive heart failure; CNS, central nervous system; IQR, interquartile range; NSAID, nonsteroidal anti-inflammatory drug; SD, standard deviation; SNF, skilled nursing facility.

* Race missing in $3(0.2 \%)$ of readmitted patients and $64(0.8 \%)$ of patients not readmitted.

${ }^{\dagger}$ Ethnicity missing in 25 (1.4\%) of readmitted patients and 327 (3.8\%) of patients not readmitted.

${ }^{4}$ Language missing in 363 (19.8\%) of readmitted patients and 1445 (17.0\%) of patients not readmitted.

${ }^{\S}$ High-risk medications charged in last 48 hours of stay.

\section{TABLE 2. Factors Associated With Readmission Within}

\section{Days}

\begin{tabular}{lll} 
Covariate & Unadjusted 0R $(\mathbf{9 5 \%}$ CI) & Adjusted 0R (95\% CI) \\
\hline Age & $1.00(0.99-1.00)$ & $1.00(0.99-1.00)$ \\
Race & & \\
$\quad$ White & Referent & Referent \\
Black & $1.67(1.47-1.91)$ & $1.43(1.24-1.65)$ \\
Asian & $0.99(0.86-1.14)$ & $0.95(0.82-1.11)$ \\
Other & $0.89(0.76-1.06)$ & $0.84(0.67-1.06)$ \\
Payer & & \\
Medicare & Referent & Referent \\
Medicaid/medi-cal & $1.37(1.21-1.55)$ & $1.15(0.97-1.36)$ \\
Private & $0.83(0.73-0.95)$ & $0.78(0.65-0.95)$ \\
Other & $0.21(0.14-0.30)$ & $0.23(0.11-0.45)$ \\
Disposition & & \\
To home & Referent & Referent \\
SNF & $1.02(0.85-1.21)$ & $0.98(0.82-1.18)$ \\
Other & $0.58(0.48-0.70)$ & $0.53(0.43-0.66)$ \\
High-risk medications & & \\
Corticosteroids & $1.31(1.16-1.48)$ & $1.24(1.09-1.42)$ \\
Narcotics & $1.49(1.34-1.65)$ & $1.33(1.16-1.53)$ \\
Anticholinergics & $0.64(0.47-0.87)$ & $0.66(0.48-0.90)$ \\
Comorbidities & & \\
Congestive heart failure & $1.39(1.19-1.63)$ & $1.30(1.09-1.56)$ \\
Neurological disorders & $0.69(0.56-0.86)$ & $0.70(0.57-0.87)$ \\
Renal failure & $1.35(1.19-1.55)$ & $1.19(1.05-1.36)$ \\
Metastatic cancer & $1.52(1.26-1.83)$ & $1.61(1.33-1.95)$ \\
Solid tumor w/o metastasis & $1.81(1.43-2.29)$ & $1.95(1.54-2.47)$ \\
Deficiency anemia & $1.41(1.26-1.58)$ & $1.27(1.13-1.44)$ \\
Weight loss & $1.30(1.08-1.57)$ & $1.26(1.09-1.47)$ \\
\hline
\end{tabular}

NOTE: Clustered by admitting MD.

Abbreviations: CI, confidence interval; OR, odds ratio; SNF, skilled nursing facility.

Several of the strongest associations, and perhaps most conducive to targeted interventions, were high-risk medications at discharge. Risk related to medications and adverse drug events following discharge have been a consistent theme in readmission literature. ${ }^{24,33}$ Our current system, which includes mandated inpatient medicine reconciliation, does not encourage discontinuation of unnecessary medica- tions to combat polypharmacy, address affordability of medications, provide consistent medication counseling, or focus on the highest risk medications. In fact, bundled interventions which implement pharmacists to focus on these measures have been successful in decreasing readmission, ${ }^{14,16,29}$ but unfortunately are not yet part of the standard of care. The challenge remains transforming a mandatory policy such as medicine reconciliation into a valuable and systematic tool in the discharge process.

Two factors were surprisingly protective against readmission: neurologic diagnosis and anticholinergic medications. This first may be explained by the presence of a separate neurology service at our institution which skews our data. For example, a patient with acute stroke, who has a $20 \%$ rate of bounce-back to a higher level of care within 30 days of discharge, ${ }^{34}$ would be admitted to the neurology service, not general medicine, and therefore would not $\mathrm{nr}$ part of our cohort. Regarding anticholinergics, several factors may explain this unexpected result. First, use of anticholinergics was relatively rare in our sample $(2.7 \%$ in readmitted patients, $4.1 \%$ in patients not readmitted), possibly creating a false positive result. Second, Hanlon et al. ${ }^{24}$ showed only a weak association at best between anticholinergics and postdischarge adverse drug reactions (hazard ratio, 1.11; 95\% CI, 0.86-1.43). Lastly, anticholinergics include a varied group of medications, therefore diluting possible relative risk of specific medications.

While this study allows providers to identify patients at increased risk of readmission, the identified factors do not fully account for readmission risk; we did not aim to produce a risk-prediction rule with our study. Prior readmission studies have been unable to create a tool to predict which patients will be readmitted with much success. ${ }^{35-37}$ These results underscore the complexities and variability of readmission, which often lack a clear single cause and effect relationship. Given the breadth of risk factors we identified, it seems likely that more intensive interventions will require a multidisciplinary approach, one which might be costly if applied broadly. Our study does not attempt to predict who will be readmitted and who will not, but rather provides a 
list of risk factors which might be used to deploy resources more efficiently.

This study had several limitations. We did not capture readmissions to outside hospitals, which account for $22 \%$ to $24 \%$ of all readmissions in prior studies, and therefore have underestimated the readmission rate in our population., ${ }^{2,8}$ However, by limiting our data to 2 hospitals within 1 institution, we were able to include more detailed patient level data, which is not accurately available in other large databases. Also, while studies of risk factors in a managed care population (such as within Medicare, the Veterans Affairs medical centers, or countries with national integrated medical records) are able to capture all readmissions, this study is the first to evaluate readmissions risk factors in a truly heterogeneous U.S. inpatient medicine population without limitation by age or payer status. Second, we did not have access to outpatient medications lists; however use of these same medications within the last 48 hours of admission is likely a reasonable proxy for outpatient use and more conducive to potential interventions (such as medication reconciliation or patient education) that could flag patients prior to discharge. Payer data was limited to only the primary payer, so patients who were dual eligible (ie, have both Medicare and Medicaid) were categorized as Medicare. Regarding sociodemographic factors, while primary language other than English was not associated with readmission, language data was missing in $17.4 \%$ of admissions, thereby limiting our ability to evaluate this factor. Our data did not include access to outpatient or primary care, and therefore we were unable to evaluate access to postdischarge follow-up care as a risk factor for readmission. Lastly, while this study did not include outpatient deaths, we did exclude patients who died in the hospital.

\section{Conclusions}

Readmission is common among general medicine patients, with approximately 1 in 5 patients being readmitted within 30 days. While the identified associated factors do not account for all the potential reasons for readmission, our study suggests a spectrum of risk factors which might be used to target more intensive multidisciplinary interventions. Specifically, the nonclinical factors of race and payer status merit further in depth research incorporating the patient experience to truly determine causation of readmission. Hospitalists, who are at nexus of the discharge process and uniquely invested in quality inpatient care, are ideally positioned to lead efforts to reduce readmissions. How to use our study's results to develop and implement effective interventions to reduce readmissions remains a subject for future studies.

\section{Address for correspondence and reprint requests:}

Nazima Allaudeen, MD, Department of Medicine, Veterans Affairs (VA) Healthcare System, 3801 Miranda Ave, MC 111, Palo Alto, CA, 94304; Telephone: 650-493-5000; Fax: 650-849-1213; E-mail: nazima.allaudeen@va.gov Received 25 August 2009; revision received 16 November 2009; accepted 3 May 2010.

\section{References}

1. A path to bundled payment around a rehospitalization.: Medicare payment Advisory Commission; June 2005.

2. Jencks SF, Williams MV, Coleman EA. Rehospitalizations among patients in the Medicare fee-for-service program. N Engl J Med. 2009;360(14): 1418-1428.

3. University HealthSystem Consortium. Available at: https://www.uhc.edu. Accessed May 2010.

4. U.S. Department of Health \& Human Services. Available at: http:// www.hospitalcompare.hhs.gov. Accessed May 2010.

5. A New Era of Responsibility: Renewing America's Promise. Available at: http://www.gpoaccess.gov/usbudget/fy10/pdf/fy10-newera.pdf. Accessed May 2010.

6. Dobrzanska L, Newell R. Readmissions: a primary care examination of reasons for readmission of older people and possible readmission risk factors. J Clin Nurs. 2006;15(5):599-606.

7. Donnan PT, Dorward DW, Mutch B, Morris AD. Development and validation of a model for predicting emergency admissions over the next year (PEONY): a UK historical cohort study. Arch Intern Med. 2008;168(13): 1416-1422.

8. Laniece I, Couturier P, Drame M, et al. Incidence and main factors associated with early unplanned hospital readmission among French medical inpatients aged 75 and over admitted through emergency units. Age Ageing. 2008;37(4):416-422.

9. Marcantonio ER, McKean S, Goldfinger M, Kleefield S, Yurkofsky M, Brennan TA. Factors associated with unplanned hospital readmission among patients 65 years of age and older in a Medicare managed care plan. Am J Med. 1999;107(1):13-17.

10. Reed RL, Pearlman RA, Buchner DM. Risk factors for early unplanned hospital readmission in the elderly. J Gen Intern Med. 1991;6(3):223-228.

11. Smith DM, Giobbie-Hurder A, Weinberger M, et al. Predicting non-elective hospital readmissions: a multi-site study. Department of Veterans Affairs Cooperative Study Group on Primary Care and Readmissions. J Clin Epidemiol. 2000;53(11):1113-1118.

12. Howie-Esquivel J, Dracup K. Effect of gender, ethnicity, pulmonary disease, and symptom stability on rehospitalization in patients with heart failure. Am J Cardiol. 2007;100(7):1139-1144.

13. Coleman EA, Parry C, Chalmers S, Min SJ. The care transitions intervention: results of a randomized controlled trial. Arch Intern Med. 2006; 166(17):1822-1828.

14. Dudas V, Bookwalter T, Kerr KM, Pantilat SZ. The impact of follow-up telephone calls to patients after hospitalization. Am J Med. 2001;111(9B): 26S-30S.

15. Jack BW, Chetty VK, Anthony D, et al. A reengineered hospital discharge program to decrease rehospitalization: a randomized trial. Ann Intern Med. 2009;150(3):178-187.

16. Koehler BE, Richter KM, Youngblood L, et al. Reduction of 30-day postdischarge hospital readmission or emergency department (ED) visit rates in high-risk elderly medical patients through delivery of a targeted care bundle. J Hosp Med. 2009;4(4):211-218.

17. Phillips CO, Wright SM, Kern DE, Singa RM, Shepperd S, Rubin HR. Comprehensive discharge planning with postdischarge support for older patients with congestive heart failure: a meta-analysis. JAMA. 2004; 291(11):1358-1367.

18. Stewart S, Horowitz JD. Home-based intervention in congestive heart failure: long-term implications on readmission and survival. Circulation. 2002;105(24):2861-2866.

19. Riegel B, Carlson B, Kopp Z, LePetri B, Glaser D, Unger A. Effect of a standardized nurse case-management telephone intervention on resource use in patients with chronic heart failure. Arch Intern Med. 2002;162(6):705-712.

20. Sin DD, Bell NR, Svenson LW, Man SF. The impact of follow-up physician visits on emergency readmissions for patients with asthma and chronic 
obstructive pulmonary disease: a population-based study. Am J Med. 2002;112(2):120-125.

21. Burns R, Nichols LO. Factors predicting readmission of older general medicine patients. J Gen Intern Med. 1991;6(5):389-393.

22. BOOSTing Care Transitions. Available at: http://www.hospitalmedicine. org/ResourceRoomRedesign/RR_CareTransitions/CT_Home.cfm. Accessed May 2010.

23. Forster AJ, Murff HJ, Peterson JF, Gandhi TK, Bates DW. Adverse drug events occurring following hospital discharge. J Gen Intern Med. 2005; 20(4):317-323.

24. Hanlon JT, Pieper CF, Hajjar ER, et al. Incidence and predictors of all and preventable adverse drug reactions in frail elderly persons after hospital stay. J Gerontol A Biol Sci Med Sci. 2006;61(5):511-515.

25. Hughes J. Development of the $3 \mathrm{M}^{\mathrm{TM}}$ All Patient Refined Diagnosis Related Groups (APR DRGs). Available at: http://www.ahrq.gov/qual/ mortality/Hughes.htm. Accessed May 2010.

26. Elixhauser A, Steiner C, Fraser I. Volume thresholds and hospital characteristics in the United States. Health Aff (Millwood). 2003;22(2): 167-177.

27. Kralovec PD, Miller JA, Wellikson L, Huddleston JM. The status of hospital medicine groups in the United States. J Hosp Med. 2006;1(2): 75-80.

28. Kuo YF, Sharma G, Freeman JL, Goodwin JS. Growth in the care of older patients by hospitalists in the United States. N Engl J Med. 2009;360(11): 1102-1112.

29. Schnipper JL, Kirwin JL, Cotugno MC, et al. Role of pharmacist counseling in preventing adverse drug events after hospitalization. Arch Intern Med. 2006;166(5):565-571.
30. Counsell SR, Holder CM, Liebenauer LL, et al. Effects of a multicomponent intervention on functional outcomes and process of care in hospitalized older patients: a randomized controlled trial of Acute Care for Elders (ACE) in a community hospital. J Am Geriatr Soc. 2000;48(12): 1572-1581.

31. Woodend AK, Sherrard H, Fraser M, Stuewe L, Cheung T, Struthers C. Telehome monitoring in patients with cardiac disease who are at high risk of readmission. Heart Lung. 2008;37(1):36-45.

32. Strunin L, Stone M, Jack B. Understanding rehospitalization risk: can hospital discharge be modified to reduce recurrent hospitalization? J Hosp Med. 2007;2(5):297-304.

33. Forster AJ, Murff HJ, Peterson JF, Gandhi TK, Bates DW. The incidence and severity of adverse events affecting patients after discharge from the hospital. Ann Intern Med. 2003;138(3):161-167.

34. Kind AJ, Smith MA, Frytak JR, Finch MD. Bouncing back: patterns and predictors of complicated transitions 30 days after hospitalization for acute ischemic stroke. J Am Geriatr Soc. 2007;55(3):365-373.

35. Billings J, Dixon J, Mijanovich T, Wennberg D. Case finding for patients at risk of readmission to hospital: development of algorithm to identify high risk patients. BMJ. 2006;333(7563):327.

36. Boult C, Dowd B, McCaffrey D, Boult L, Hernandez R, Krulewitch H. Screening elders for risk of hospital admission. J Am Geriatr Soc. 1993; 41(8):811-817.

37. Howell S, Coory M, Martin J, Duckett S. Using routine inpatient data to identify patients at risk of hospital readmission. BMC Health Serv Res. 2009;9:96 